\title{
High-temperature thermal expansion and structural behaviour of stromeyerite, $\mathrm{AgCuS}$
}

\author{
D M Trots ${ }^{1,2}$, A Senyshyn ${ }^{1,3}$, D A Mikhailova ${ }^{1}$, M Knapp ${ }^{4}, \mathbf{C}$ Baehtz $^{2,5}$, \\ M Hoelzel ${ }^{1,3}$ and H Fuess ${ }^{1}$ \\ ${ }^{1}$ Fachbereich Materialwissenschaft, Technische Universität Darmstadt, Petersenstrasse 23, \\ D-64287 Darmstadt, Germany \\ ${ }^{2}$ Hamburger Synchrotronstrahlungslabor am Deutsches Elektronen-Synchrotron, Notkestrasse 85, \\ D-22607 Hamburg, Germany \\ ${ }^{3}$ Forschungsneutronenquelle Heinz-Maier-Leibnitz (FRM II), Technische Universität München, \\ Lichtenbergstrasse 1, D-85747 Garching b. München, Germany \\ ${ }^{4}$ CELLS, POB 68, 08193 Barcelona, Spain \\ ${ }^{5}$ Department of Structural Diagnostics, Research Center Dresden-Rossendorf, Bautzner \\ Landstrasse 128, D-01328 Dresden, Germany \\ E-mail: d_trots@yahoo.com
}

Received 21 November 2006, in final form 12 February 2007

Published 12 March 2007

Online at stacks.iop.org/JPhysCM/19/136204

\begin{abstract}
Results of simultaneous thermal analysis, synchrotron and neutron powder diffraction in the range from room temperature up to the melting point at $936 \mathrm{~K}$ on non-superionic orthorhombic $\beta-\mathrm{AgCuS}$ as well as on superionic hexagonal $\alpha$ - and cubic $\delta$-AgCuS are reported. On heating the sample is only stable in argon. The following phase transitions occur in $\mathrm{AgCuS}$ at elevated temperatures: $\beta \stackrel{361 \mathrm{~K}}{\longrightarrow} \alpha \stackrel{399 \mathrm{~K}}{\longrightarrow} \alpha+\delta \stackrel{439 \mathrm{~K}}{\longrightarrow} \delta$. The volume changes at the superionic $\beta \longrightarrow \alpha$ and $\alpha \longrightarrow \delta$ phase transitions are about 2.3 and $0.6 \%$. The volume thermal expansion coefficients are $26 \times 10^{-6}, 130 \times 10^{-6}$ and $85 \times 10^{-6} \mathrm{~K}^{-1}$ for the pure $\beta$-, $\alpha$ - and $\delta$-phases, respectively. Models for the average structures of $\alpha$ - and $\delta$ - $\mathrm{AgCuS}$ are proposed and discussed. Ionic conductivity in $\delta$ - $\mathrm{AgCuS}$ may originate from cation jumps in 'skewed' $\langle 100\rangle$ directions between nearest-neighbour tetrahedral sites via the peripheries of the octahedral cavities. A correlation between the temperature dependence of the cation redistribution in $\delta$ - $\mathrm{AgCuS}$ and the temperature dependence of the ionic conductivity is assumed. A two-dimensional nature of the ionic conductivity due to cation jumps in slabs perpendicular to the $c$-direction is supposed for $\alpha$-AgCuS. There is no evidence for ionic diffusion through the $\left(\frac{1}{2}, \frac{1}{2}, \frac{1}{2}\right)$ site in $\langle 111\rangle$ directions in either superionic $\alpha$ - or $\delta$-phases.
\end{abstract}

\section{Introduction}

For a long time, copper and silver based chalcogenides have attracted considerable attention due to their promising ion transport properties $[1,2]$. Recently, the high ionic conductivity for 
cations renewed the interest in silver copper sulfide, $\mathrm{AgCuS}$ (mineral name stromeyerite). The ionic conductivity in the superionic face-centred cubic (fcc) phase of $\mathrm{AgCuS}$ was measured in the temperature range $473-673 \mathrm{~K}$ and has a value of $2.38 \Omega^{-1} \mathrm{~cm}^{-1}$ at $573 \mathrm{~K} \mathrm{[3]}$.

The earliest structural investigations reported an orthorhombic structure for $\mathrm{AgCuS}$ at room temperature (RT), with space group $C m c 2_{1}$ or $\mathrm{Cmcm}$ [4] and $\mathrm{Cmcm}$ [5] with lattice parameters $a=4.06 \AA, b=6.66 \AA, c=7.99 \AA$ and with four formula units per cell, $Z=4$. The RT structure was described as zigzag chains of silver and sulfur atoms parallel to the $c$ axis; the planes parallel to (001) are composed of layers of loosely packed face-centred silver atoms alternating with layers of triangularly coordinated sulfur and copper atoms [4, 5]. Shortly thereafter, phase relations in the $\mathrm{Cu}-\mathrm{Ag}-\mathrm{S}$ system were studied by Djurle [6] and Skinner [7]. Djurle investigated $\mathrm{AgCuS}$ by powder x-ray diffraction at a limited number of temperatures and reported the existence of high-temperature hexagonal and cubic modifications as well as an approximated temperature for borders of these phase regions [6]. The work of Skinner [7] was guided by mineralogical interest in stromeyerite. The following phase transitions were found: orthorhombic $\stackrel{366 \mathrm{~K}}{\longrightarrow}$ hexagonal $\stackrel{400 \mathrm{~K}}{\longrightarrow}$ hexagonal + cubic $\stackrel{438 \mathrm{~K}}{\longrightarrow}$ cubic.

Stromeyerite was studied by powder and single-crystal diffraction in more recent structural investigations of Skarda et al [8] and Baker et al [9]. The high-temperature neutron powder diffraction revealed a hexagonal phase of $\mathrm{AgCuS}$ at $388 \mathrm{~K}$ which is isostructural to $\mathrm{Cu}_{2} \mathrm{~S}$. The hexagonal AgCuS exhibits the symmetry $P 6_{3} / m m c$ with $a=4.1389(10) \AA, c=$ 7.0817(27) $\AA$ and $Z=2$ : anions occupy 2(d) and cations $12(\mathrm{k})$ sites, a partial population of 2(b) sites solely occupied by copper cations was also described [8]. The RT crystal structure of stromeyerite has been accurately determined by single-crystal $\mathrm{x}$-ray diffraction in conjunction with convergent-beam electron diffraction [9]. The primary structural features are the same as those proposed in $[4,5]$; the structure is non-centrosymmetric (space group $C m c 2_{1}$ ), polar in the $c$-direction and possesses a distorted hexagonal close packing (hcp) of sulfur. Moreover, the symmetry of stromeyerite reduces from $C m c 2_{1}$ to its maximal non-isomorphic subgroup $P m c 2_{1}$ below $250 \mathrm{~K}$ [9].

Despite a number of structural investigations on stromeyerite in the literature, no information regarding high-temperature thermal expansion and structural behaviour in the superionic state have been found. From the results presented in [4-9] a more detailed consideration of the high-temperature cubic modification of stromeyerite is recommended. Besides, more accurate in situ powder diffraction experiments on stromeyerite are required for further theoretical and experimental investigations of this highly disordered system. The present paper is therefore devoted to the investigation of $\mathrm{AgCuS}$ in a wide temperature range by simultaneous thermal analysis (STA) and powder diffraction.

\section{Experimental details}

The AgCuS sample was synthesized via a solid-state route. Preliminary x-ray diffraction measurements were performed using an STOE STADI P diffractometer $\left(\mathrm{Cu} \mathrm{K} \alpha_{1}\right.$ radiation, curved $\mathrm{Ge}(111)$ monochromator, transmission mode, step size $0.03^{\circ}(2 \theta)$, curved positionsensitive detector). In order to characterize the stability of AgCuS, STA measurements, which combined thermogravimetry (TG) and differential scanning calorimetry (DSC), were carried out on a Netzsch STA 429 device at a heating rate of $10 \mathrm{~K} \mathrm{~min}^{-1}$, either in air or in argon.

In situ high-temperature structural studies in the temperature range RT-693 K were performed at the synchrotron facility HASYLAB/DESY (Hamburg, Germany) with the powder diffractometer at beam-line B2 [10]. A $0.3 \mathrm{~mm}$ quartz capillary was filled under argon atmosphere with powdered $\mathrm{AgCuS}$ and sealed. Subsequently the capillary was 
mounted inside a STOE furnace in Debye-Scherrer geometry, equipped with a Eurotherm temperature controller and a capillary spinner. The furnace temperature was measured by a $\mathrm{Ni} / \mathrm{CrNi}$ thermocouple and calibrated using the thermal expansion of $\mathrm{NaCl}$ [11]. The wavelength of $0.49962 \AA$ was selected using a Si(111) double flat-crystal monochromator and determined from ten reflection positions of $\mathrm{LaB}_{6}$ reference material (NIST SRM 660a). All 26 diffraction patterns have been collected at fixed temperatures during the heating cycle using an image-plate detector [12] $\left(2 \theta\right.$ range $4^{\circ}-45^{\circ}$, step size $\left.0.004^{\circ}\right)$. One additional pattern was taken after the heat treatment at RT.

High-temperature neutron diffraction data were collected at the research neutron reactor FRM-II (Garching near Munich, Germany) at the structure powder diffractometer SPODI [13]. A cylindrical can of $8 \mathrm{~mm}$ diameter made from thin $(0.1 \mathrm{~mm})$ niobium foil was filled with the powder sample and mounted in a high-temperature vacuum furnace equipped with a niobium heating element. The temperature was measured by a W/Re thermocouple and controlled with a Eurotherm device. A monochromator take-off angle of $155^{\circ}$ was selected for the vertically focusing composite monochromator and the wavelength was determined to $1.5487 \AA$ by Rietveld refinement of a diffraction pattern of the silicon reference material NIST SRM 640b. Full patterns were collected over a $2 \theta$ range of $8^{\circ}-160^{\circ}$ with a step size of $0.05^{\circ}$ at $\mathrm{RT}$, 460, 478, 640, 785 and $890 \mathrm{~K}$.

Data evaluation was performed using the package 'FullProf' [14]: lattice parameters, zero shift, background (linear interpolation between selected data points in non-overlapping regions) and profile shape parameters (Caglioti) were refined by a structure independent whole-pattern decomposition procedure; the averaged structure was then analysed by the full-profile Rietveld method.

\section{Results and discussion}

\subsection{Stability of stromeyerite}

Preliminary conventional x-ray diffraction measurements revealed no impurities in the sample. $\mathrm{AgCuS}$ was then examined by STA (figure 1). The DSC curve of AgCuS measured in air displays a strong endothermic signal with its maximum at $370 \mathrm{~K}$, which corresponds to the orthorhombic $\longrightarrow$ hexagonal phase transition. This is in agreement with our diffraction studies $(361 \mathrm{~K})$ as well as with data from [6-8]. An additional weak endothermic effect with a maximum at about $405 \mathrm{~K}$ could be associated with the low-temperature boundary of the two-phase hexagonal-cubic region, corresponding well with our (399 K) and Skinners's (400.5 K) [7] value from diffraction measurements. During heating in air the TG curve clearly indicates a change of the sample weight above $535 \mathrm{~K}$, which is related to a decomposition of the sample. On the other hand, no obvious changes of the sample weight were observed in the TG curve measured under argon. X-ray diffraction analysis of the same sample also showed no changes with respect to the initial state. The DSC heating curve of $\mathrm{AgCuS}$ in argon exhibits in addition a strong endothermic signal with its maximum at $936 \mathrm{~K}$, indicating the melting of the sample.

\subsection{Structural behaviour of stromeyerite in the superionic state}

The analysis of the structural behaviour of $\mathrm{AgCuS}$ showed the following phase boundaries at elevated temperatures: orthorhombic $\left(C m c 2_{1}\right) \stackrel{361 \mathrm{~K}}{\longrightarrow}$ hexagonal $\left(P 6_{3} / m m c\right) \stackrel{399 \mathrm{~K}}{\longrightarrow}$ hexagonal $\left(P_{3} / m m c\right)$ plus cubic $(F m \overline{3} m) \stackrel{439 \mathrm{~K}}{\longrightarrow}$ cubic $(F m \overline{3} m)$. A large decrease in peak intensity after the orthorhombic $\left(C m c 2_{1}\right) \stackrel{361 \mathrm{~K}}{\longrightarrow}$ hexagonal $\left(P 6_{3} / m m c\right)$ phase transformation indicates 


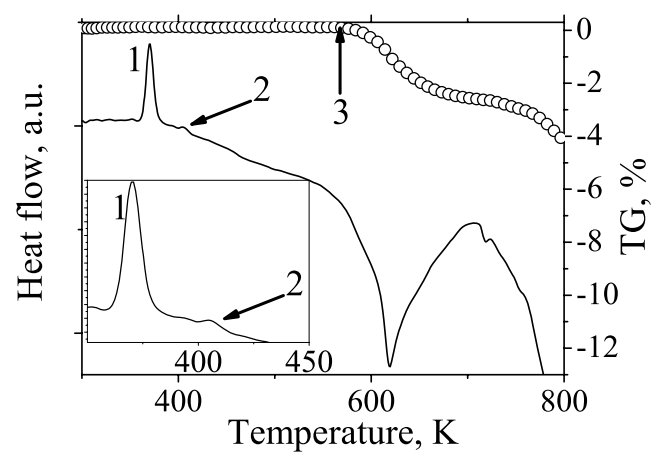

(a) STA in air

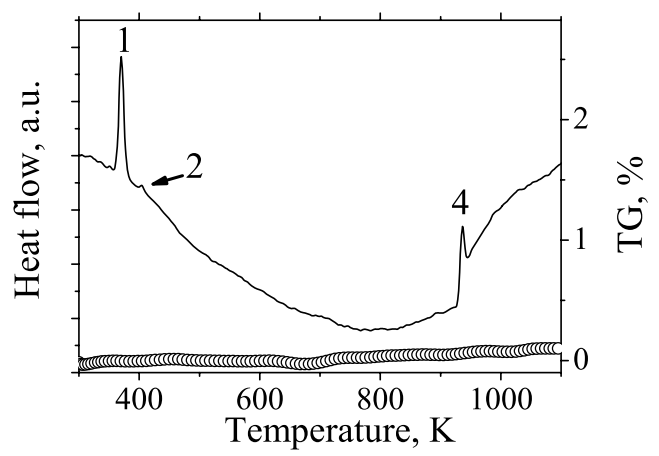

(b) STA in argon

Figure 1. STA results for $\mathrm{AgCuS}$ at a heating rate of $10 \mathrm{~K} \mathrm{~min}^{-1}$. Solid line-DSC curve, open symbols-TG curve. 1 - peak which corresponds to the orthorhombic $\rightarrow$ hexagonal phase transition; 2-weak peak which is associated with the low-temperature boundary of the two-phase hexagonal-cubic region; 3 - temperature threshold for the sample decomposition in air; 4-melting point.

a high degree of structural disorder introduced by transition. The temperatures of the phase boundaries are in good agreement with the results presented in [7] but, nevertheless, the structural modifications of stromeyerite were insufficiently described in this reference. The analysis of the diffraction pattern from the sample after heat treatment in argon during the diffraction measurements showed no changes with respect to the initial state.

In the newest work on the structure of stromeyerite by Baker et al [9], for convenience, following the analogy with silver iodide, the low-temperature phase was designated as $\gamma$ $\mathrm{AgCuS}$, the RT phase as $\beta$-AgCuS, and the cation-conducting high-temperature phase as $\alpha$ $\mathrm{AgCuS}$. Nevertheless, it is not clear whether the hexagonal or the cubic high-temperature phase was denoted as $\alpha$-AgCuS by Baker et al. To clarify this matter by following the notation accepted for other superionic conductors (see for instance [15]), the hexagonal structure of $\mathrm{AgCuS}$ should be denoted as $\alpha-\mathrm{AgCuS}$ and the cubic phase at the temperature immediately above the $\alpha$-phase should be denoted as $\delta$-phase. Results of Rietveld refinements for selected temperatures for $\beta$-, $\alpha$ - and $\delta$ - $\mathrm{AgCuS}$ are illustrated in figure 2 and summarized in tables $1-3$.

3.2.1. $\alpha-A g C u S$. As was recently proposed in [8], the structural model for hexagonal $\mathrm{AgCuS}$ was based on the structural model of $\mathrm{Cu}_{2} \mathrm{~S}$ determined by single-crystal neutron diffraction [16]. Hence, the sulfur ions of $\alpha-\mathrm{AgCuS}$ adopt an hep structure on the 2(d) sites. At 


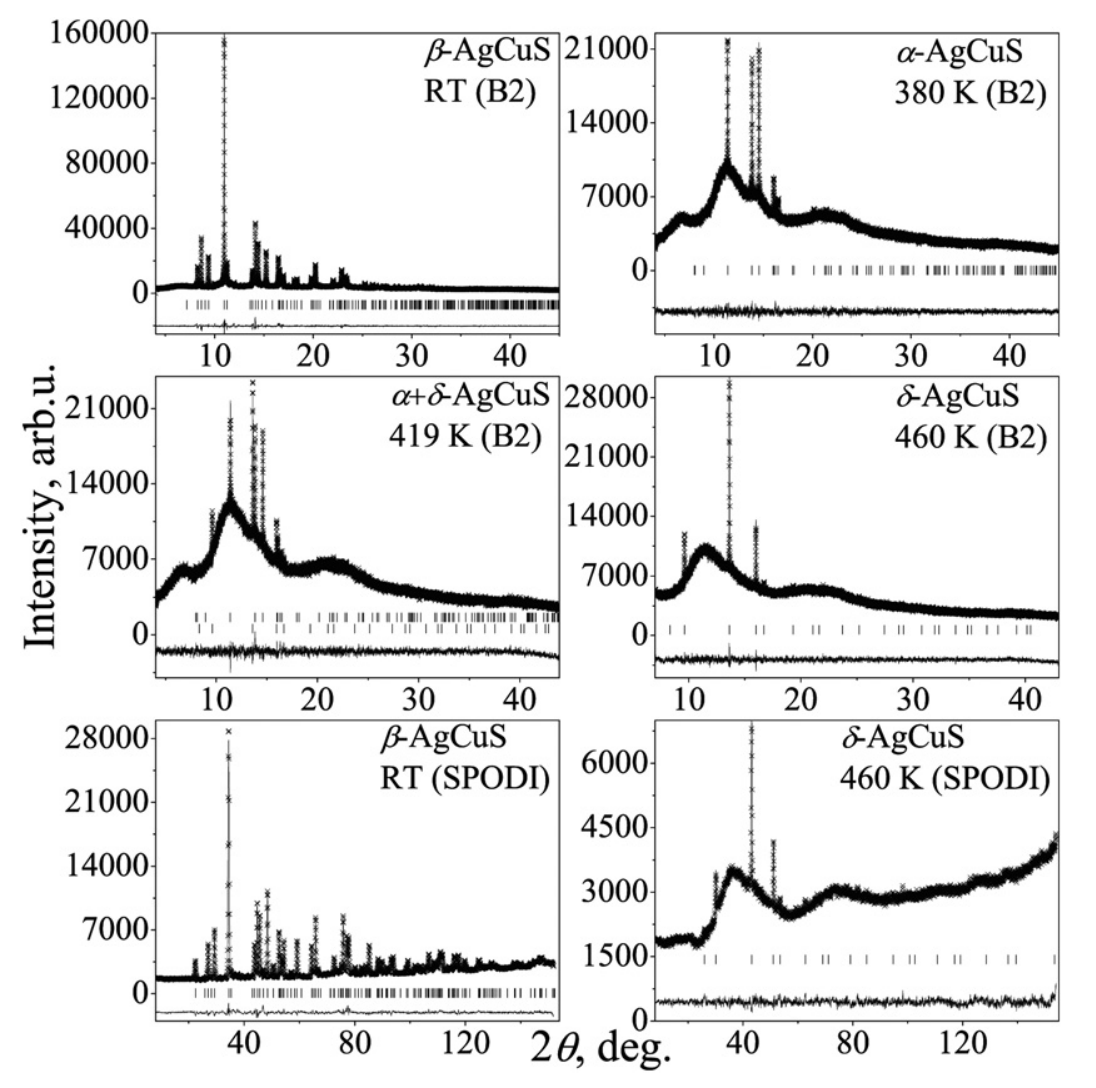

Figure 2. Results of Rietveld refinements for data collected with synchrotron (B2) and neutron (SPODI) diffractometers. Crosses are experimental data; the line through the crosses is the calculated profile and the lower curve their difference. Tick marks show the calculated positions of $\mathrm{AgCuS}$ reflections.

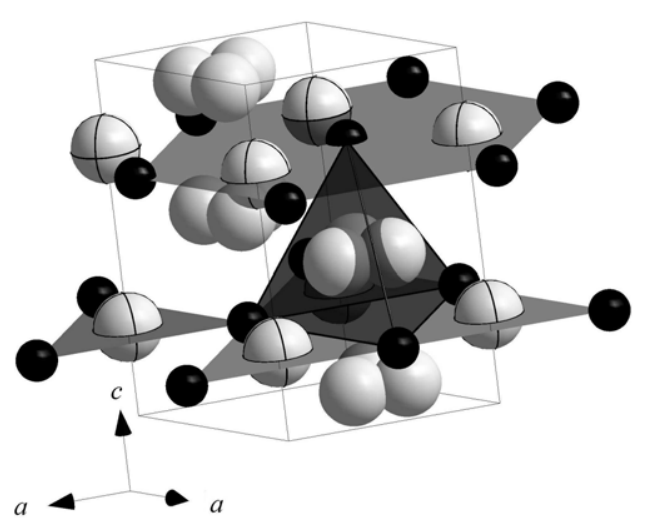

Figure 3. A schematic diagram showing the average structure of hexagonal $\alpha$-AgCuS at $380 \mathrm{~K}$. Black spheres, grey spheres and grey spheres with inner lines- $\mathrm{S}^{2-}$ in $2(\mathrm{~d}), \mathrm{Ag}^{+} / \mathrm{Cu}^{+}$in triangular $12(\mathrm{k})$ and $\mathrm{Cu}^{+}$in triangular 2(b) sites, correspondingly. $100 \%$ occupancy of all crystallographic positions is illustrated. 
Table 1. Structural parameters of orthorhombic $(\beta)$ and hexagonal $(\alpha) \operatorname{AgCuS}$. Experimental data were collected with the B2 diffractometer. $\chi^{2}$ is the goodness of fit. The isotropic thermal parameters $u_{\text {iso }}$ of cations and copper occupancy in 2(b) and 12(k) positions in $\alpha$-AgCuS were constrained during refinements. Positional parameters of silver and copper in $12(\mathrm{k})$ positions were constrained as well (at $y=2 x$ ).

\begin{tabular}{|c|c|c|}
\hline & $C m c 2_{1}, Z=4, \mathrm{RT}$ & $P 6_{3} / m m c, Z=2,380 \mathrm{~K}$ \\
\hline$a(\AA)$ & $4.0633(2)$ & $4.1434(5)$ \\
\hline$b(\AA)$ & $6.6281(4)$ & - \\
\hline$c(\AA)$ & $7.9713(4)$ & $7.0813(8)$ \\
\hline $\mathrm{S}$, site & 4(a) & $2(d)$ \\
\hline$x$ & $\frac{1}{2}$ & $\frac{1}{3}$ \\
\hline$y$ & $0.287(2)$ & $\frac{2}{3}$ \\
\hline$z$ & $0.259(8)$ & $\frac{3}{4}$ \\
\hline$u_{\text {iso }}\left(\AA^{2}\right)$ & $0.020(2)$ & $0.089(1)$ \\
\hline Occupancy (\%) & 100 & 100 \\
\hline Ag, site & 4(a) & $12(\mathrm{k})$ \\
\hline$x$ & $\frac{1}{2}$ & $0.246(1)$ \\
\hline$y$ & 0.495 & $0.492(2)$ \\
\hline$z$ & $\frac{1}{2}$ & $0.436(1)$ \\
\hline$u_{\text {iso }}\left(\AA^{2}\right)$ & $0.0787(13)$ & $0.200(8)$ \\
\hline Occupancy (\%) & 100 & 16.7 \\
\hline $\mathrm{Cu}$, site & 4(a) & $12(\mathrm{k})$ \\
\hline$x$ & 0 & $0.246(1)$ \\
\hline$y$ & $0.432(1)$ & $0.492(2)$ \\
\hline$z$ & $0.248(2)$ & $0.436(1)$ \\
\hline$u_{\text {iso }}\left(\AA^{2}\right)$ & $0.0287(15)$ & $0.200(8)$ \\
\hline Occupancy (\%) & 100 & $4.1(8)$ \\
\hline $\mathrm{Cu}$, site & - & 2(b) \\
\hline$x$ & - & 0 \\
\hline$y$ & - & 0 \\
\hline$z$ & - & $\frac{1}{4}$ \\
\hline$u_{\text {iso }}\left(\AA^{2}\right)$ & - & $0.200(8)$ \\
\hline Occupancy (\%) & - & $75.4(8)$ \\
\hline$\chi^{2}$ & 8.8 & 9.0 \\
\hline
\end{tabular}

$380 \mathrm{~K}$ about $75 \%$ of all copper resides in triangular coordination within these hep sulfur layers partially occupying 2(b) sites, whereas about $25 \%$ of the copper and all silver partially occupy the triangularly coordinated $12(\mathrm{k})$ sites (see figure 3 and table 1). The cations in the 12(k) sites at $(x, 2 x, z)$ with $x=0.246(1)$ and $z=0.436(1)$ at $380 \mathrm{~K}$ are slightly displaced from the faces of the triangular surroundings towards the centres of the distorted sulfur tetrahedra (possible variants of such triangular surroundings and tetrahedra can be seen in figure 3 ). The temperature dependences of the parameters of the average structure of $\alpha$-AgCuS are presented in figure 4. Pronounced non-uniformity has been observed in the thermal behaviour of the positional parameters of the cations occupying the $12(\mathrm{k})$ site at $(x, 2 x, z)$, where $x$ increases with temperature and $z$ decreases. This leads to a modest shift of the cations towards the centres of the distorted sulfur tetrahedra (see figure 3), which correlates well with a significant cation occupancy of the tetrahedral cavities in $\delta$ - $\mathrm{AgCuS}$ (see next section). Isotropic thermal 
Table 2. A summary of the possible structural models used to interpret the diffraction data for the cubic $(\delta) \mathrm{AgCuS}$ at $460 \mathrm{~K}$. Neutron and synchrotron diffraction data were treated simultaneously. $\chi^{2}$ is goodness of fit. The isotropic thermal parameters $u_{\text {iso }}$ and positional parameters of cations as well as occupancy of cations in 'split' positions were constrained during refinements.

\begin{tabular}{llllllll}
\hline \multicolumn{7}{c}{$F m \overline{3} m, Z=4, T=460 \mathrm{~K}, a=5.9564(6) \AA$} \\
\hline Model & 1 & 2 & 3 & 4 & 5 & 6 & 7 \\
\hline $\mathrm{S}$, site & & & \multicolumn{5}{c}{$4(\mathrm{a})$ at $(0,0,0)$} \\
$u_{\text {iso }}\left(\AA^{2}\right)$ & $0.023(1)$ & $0.059(4)$ & $0.454(4)$ & $0.030(3)$ & $0.139(13)^{\mathrm{a}}$ & $0.197(16)$ & $0.127(20)$ \\
Occupancy $(\%)$ & 100 & 100 & 100 & 100 & 100 & 100 & 100 \\
\hline $\mathrm{Ag} / \mathrm{Cu}$, site & $8(\mathrm{c})$ & $32(\mathrm{f})$ & $8(\mathrm{c})$ & $8(\mathrm{c})$ & $8(\mathrm{c})$ & $8(\mathrm{c})$ & $32(\mathrm{f})$ \\
$x$ & $\frac{1}{4}$ & $0.324(5)$ & $\frac{1}{4}$ & $\frac{1}{4}$ & $\frac{1}{4}$ & $\frac{1}{4}$ & $0.305(6)$ \\
$y$ & $\frac{1}{4}$ & $0.324(5)$ & $\frac{1}{4}$ & $\frac{1}{4}$ & $\frac{1}{4}$ & $\frac{1}{4}$ & $0.305(6)$ \\
$z$ & $\frac{1}{4}$ & $0.324(5)$ & $\frac{1}{4}$ & $\frac{1}{4}$ & $\frac{1}{4}$ & $\frac{1}{4}$ & $0.305(6)$ \\
$u_{\text {iso }}\left(\AA^{2}\right)$ & $0.091(1)$ & $0.304(12)$ & $0.121(7)$ & $0.333(7)$ & $0.139(13)^{\mathrm{a}}$ & $0.369(15)$ & $0.116(20)$ \\
Occupancy $(\%)$ & 100 & 25 & $68(1)$ & $63.0(9)$ & $26.8(9)$ & $25.4(3)$ & $11(3)$ \\
\hline Ag/Cu, site & - & - & $4(\mathrm{~b})$ & $24(\mathrm{~d})$ & $32(\mathrm{f})$ & $48(\mathrm{i})$ & $32(\mathrm{f})$ \\
$x$ & - & - & $\frac{1}{2}$ & 0 & $0.370(5)$ & $0.347(2)$ & $0.375(3)$ \\
$y$ & - & - & $\frac{1}{2}$ & $\frac{1}{4}$ & $0.370(5)$ & $0.347(2)$ & $0.375(3)$ \\
$z$ & - & - & $\frac{1}{2}$ & $\frac{1}{4}$ & $0.370(5)$ & $\frac{1}{2}$ & $0.375(3)$ \\
$u_{\text {iso }}\left(\AA^{2}\right)$ & - & - & $0.121(7)$ & $0.333(7)$ & $0.139(13)^{\mathrm{a}}$ & $0.369(15)$ & $0.116(20)$ \\
Occupancy $(\%)$ & - & - & $64(1)$ & $12.3(9)$ & $18.3(9)$ & $12.4(3)$ & $13(3)$ \\
\hline$\chi^{2}$ & 14.3 & 11.0 & 15.8 & 15.0 & 6.3 & 7.9 & 8.3 \\
\hline
\end{tabular}

${ }^{a}$ During refinements $u_{\text {iso }}$ of anions and cations have shown similar values at all considered temperatures; therefore, the overall thermal parameter was used in model 5.

parameters, $u_{\text {iso }}$, and the distribution of mobile cations between the two types of interstitial sites remain insensitive to temperature changes. An identical structural behaviour was reported for hexagonal copper sulfide, $\mathrm{Cu}_{2} \mathrm{~S}$ [16]. We therefore propose the same mechanism for the cation transport as reported for $\mathrm{Cu}_{2} \mathrm{~S}$ in [16]: diffusion through the $\left(\frac{1}{2}, \frac{1}{2}, \frac{1}{2}\right)$ site in the $\langle 111\rangle$ direction is insufficient; the cation conductivity instead is assumed to be two dimensional and occurs primarily in slabs perpendicular to the $c$-direction.

The trigonal coordination of copper in the distorted hcp structure of sulfur reflects the principal similarity of the hexagonal and the orthorhombic phases. The possibility of a similar coordination of the cations in the disordered conducting structure relative to their coordinations in the non-conducting phases was previously reported for $\mathrm{AgI}, \mathrm{Ag}_{2} \mathrm{~S}$ and $\mathrm{Cu}_{2} \mathrm{~S}$ [16]. Besides, the change of the silver coordination from twofold in $\beta$-AgCuS and $\gamma-\mathrm{AgCuS}$ [9] to threefold in $\alpha$-AgCuS should be noted. The cation-anion bond distances for hexagonal $\mathrm{AgCuS}$ at $380 \mathrm{~K}$ are 2.512(5) $\AA$ and 2.303(5) $\AA$ for $\mathrm{Cu} / \mathrm{Ag}-\mathrm{S}$ distances (i.e. cations in 12(k) sites) and 2.397(5) $\AA$ for $\mathrm{Cu}-\mathrm{S}$ distances (cations in 2(b) sites). This is in good agreement with the cation-anion bond distances for the orthorhombic phase, which range between $2.53-2.60 \AA$ and $2.25-2.28 \AA$ for Ag-S distances as well as 2.24-2.27 $\AA$ and 2.31-2.35 $\AA$ for $\mathrm{Cu}-\mathrm{S}$ distances.

3.2.2. $\delta$-AgCuS. The cation distribution in $\delta$-AgCuS was parameterized using different structural models with silver and copper cations randomly distributed over tetrahedral and octahedral interstitial sites within a rigid sulfur fcc sublattice. Firstly, simultaneous Rietveld refinements based on synchrotron and neutron diffraction data collected from $\delta$ - $\mathrm{AgCuS}$ at 460 
Table 3. A summary of the possible structural models used to interpret the diffraction data for the cubic $(\delta) \mathrm{AgCuS}$ at $640 \mathrm{~K}$. Neutron and synchrotron diffraction data were treated simultaneously. $\chi^{2}$ is goodness of fit. The isotropic thermal parameters $u_{\text {iso }}$ and positional parameters of cations as well as occupancy of cations in 'split' positions were constrained during refinements.

\begin{tabular}{llllllll}
\hline \multicolumn{7}{c}{$F m \overline{3} m, Z=4, T=640 \mathrm{~K}, a=5.9863(10) \AA$} \\
\hline Model & 1 & 2 & 3 & 4 & 5 & 6 & 7 \\
\hline $\mathrm{S}$, site & & & \multicolumn{5}{l}{$4(\mathrm{a})$ at $(0,0,0)$} \\
$u_{\text {iso }}\left(\AA^{2}\right)$ & $0.129(13)$ & $0.228(25)$ & $0.543(18)$ & $0.139(25)$ & $0.130(13)^{\mathrm{a}}$ & $0.241(13)$ & $0.151(12)$ \\
Occupancy $(\%)$ & 100 & 100 & 100 & 100 & 100 & 100 & 100 \\
\hline $\mathrm{Ag} / \mathrm{Cu}$, site & $8(\mathrm{c})$ & $32(\mathrm{f})$ & $8(\mathrm{c})$ & $8(\mathrm{c})$ & $8(\mathrm{c})$ & $8(\mathrm{c})$ & $32(\mathrm{f})$ \\
$x$ & $\frac{1}{4}$ & $0.338(5)$ & $\frac{1}{4}$ & $\frac{1}{4}$ & $\frac{1}{4}$ & $\frac{1}{4}$ & $0.304(9)$ \\
$y$ & $\frac{1}{4}$ & $0.338(5)$ & $\frac{1}{4}$ & $\frac{1}{4}$ & $\frac{1}{4}$ & $\frac{1}{4}$ & $0.304(9)$ \\
$z$ & $\frac{1}{4}$ & $0.338(5)$ & $\frac{1}{4}$ & $\frac{1}{4}$ & $\frac{1}{4}$ & $\frac{1}{4}$ & $0.304(9)$ \\
$u_{\text {iso }}\left(\AA^{2}\right)$ & $0.725(19)$ & $0.485(25)$ & $0.156(18)$ & $0.583(25)$ & $0.130(13)^{\mathrm{a}}$ & $0.481(12)$ & $0.089(12)$ \\
Occupancy $(\%)$ & 100 & 25 & $63(1)$ & $57.3(8)$ & $31.3(10)$ & $28.2(4)$ & $8.6(7)$ \\
\hline $\mathrm{Ag} / \mathrm{Cu}$, site & - & - & $4(\mathrm{~b})$ & $24(\mathrm{~d})$ & $32(\mathrm{f})$ & $48(\mathrm{i})$ & $32(\mathrm{f})$ \\
$x$ & - & - & $\frac{1}{2}$ & 0 & $0.369(5)$ & $0.335(2)$ & $0.384(7)$ \\
$y$ & - & - & $\frac{1}{2}$ & $\frac{1}{4}$ & $0.369(5)$ & $0.335(2)$ & $0.384(7)$ \\
$z$ & - & - & $\frac{1}{2}$ & $\frac{1}{4}$ & $0.369(5)$ & $\frac{1}{2}$ & $0.384(7)$ \\
$u_{\text {iso }}\left(\AA^{2}\right)$ & - & - & $0.156(18)$ & $0.583(25)$ & $0.130(13)^{\mathrm{a}}$ & $0.481(12)$ & $0.089(12)$ \\
Occupancy $(\%)$ & - & - & $74(1)$ & $14.2(8)$ & $17.2(10)$ & $12.0(4)$ & $16.5(7)$ \\
\hline$\chi^{2}$ & 12.4 & 11.2 & 17.6 & 10.5 & 8.1 & 10.1 & 10.1 \\
\hline
\end{tabular}

${ }^{a}$ During refinements $u_{\text {iso }}$ of anions and cations have shown similar values at all considered temperatures; therefore, the overall thermal parameter was used in model 5.

and $640 \mathrm{~K}$ were performed. Such refinements gave a more accurate model for the average structure due to the reduced number of refined structural parameters (see for example [17]). During refinement great care was taken to ensure that data were not overinterpreted by models with an inappropriate large number of structural parameters. Secondly, separate refinements were performed on the basis of synchrotron and neutron diffraction data at all temperatures measured using the model from simultaneous refinement. On the whole, the results of simultaneous as well as separate Rietveld refinements are comparable, although the moderate differences generated by the different scattering techniques and counting statistics can be observed.

Hence, simultaneous Rietveld refinements were based on a $F m \overline{3} m$ symmetry and are summarized in tables 2 and 3. The results demonstrate a considerable degree of cation disorder which can be modelled by a random occupation of the tetrahedral $8(\mathrm{c})$ site at $\left(\frac{1}{4}, \frac{1}{4}, \frac{1}{4}\right)$ and the octahedral 32(f) site at ( $x, x, x)$ with $\frac{1}{3}<x<\frac{1}{2}$ (model 5, tables 2 and 3). This model indicates cation disorder in $\langle 111\rangle$ directions. It should also be noted that at $x \sim 0.37-0.38$, as in our case, cations are practically occupying 32(f) $(x, x, x)$ sites on the boundary between tetrahedral and octahedral cavities but are noticeably shifted towards the centres of the octahedra. Therefore, we consider the 32(f) site at $(x, x, x)$ with $x \sim 0.37-0.38$ as octahedral (see figure 5). Attempts were also made to refine the data in the ideal antifluorite structure and in a 'one-site' model with cations distributed in $\langle 111\rangle$ directions in 32(f) at $(x, x, x)$ with $\frac{1}{4}<x<\frac{1}{3}$ (see tables 2 and 3 ). Furthermore, the following models with cations distributed between two sites were tested: between $8(\mathrm{c})$ sites at $\left(\frac{1}{4}, \frac{1}{4}, \frac{1}{4}\right)$ and $4(\mathrm{~b})$ at $\left(\frac{1}{2}, \frac{1}{2}, \frac{1}{2}\right)$ as well as between $32(\mathrm{f})$ at $(x, x, x)$ 


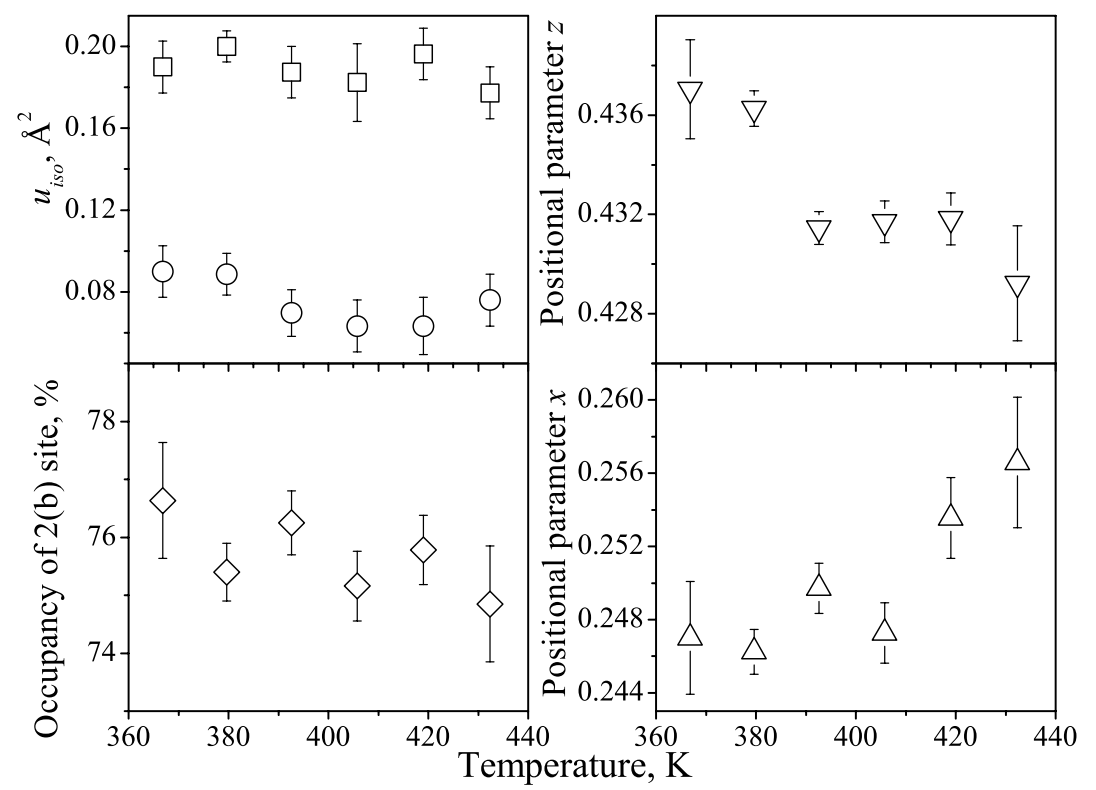

Figure 4. Temperature dependences of isotropic thermal parameters of $\mathrm{S}^{2-}$ (circles) and $\mathrm{Ag}^{+} / \mathrm{Cu}^{+}$ (squares), normalized site occupation number of $\mathrm{Cu}^{+}$residing on the triangularly coordinated 2(b) sites and cation positional parameters of $12(\mathrm{k})$ at $(x, 2 x, z)$ sites within the hexagonal $\alpha-\mathrm{AgCuS}$.

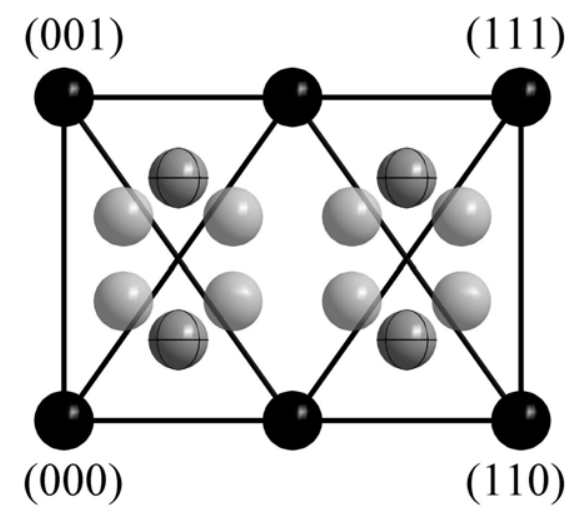

Figure 5. Schematic representation of the average structure of $\delta$ - $\mathrm{AgCuS}$ at $460 \mathrm{~K}$. The plot shows a (110) section through the unit cell. Black spheres, grey spheres with inner lines and grey spheres$\mathrm{S}^{2-}$ in the 4(a), $\mathrm{Ag}^{+} / \mathrm{Cu}^{+}$in the ideal tetrahedral 8(c) and $\mathrm{Ag}^{+} / \mathrm{Cu}^{+}$in the octahedral 32(f) sites, respectively. The plot shows $100 \%$ occupancy for all crystallographic positions. The diagonal lines indicate the positions of the boundaries between tetrahedral and octahedral interstices.

with $\frac{1}{4}<x<\frac{1}{3}$ and 32(f) at $(x, x, x)$ with $\frac{1}{3}<x<\frac{1}{2}$ (illustrating cation disorder in the $\langle 111\rangle$ direction); between $8(\mathrm{c})$ at $\left(\frac{1}{4}, \frac{1}{4}, \frac{1}{4}\right)$ and $24(\mathrm{~d})$ at $\left(0, \frac{1}{4}, \frac{1}{4}\right)$ sites (illustrating cation disorder in the $\langle 100\rangle$ direction); between $8(\mathrm{c})$ at $\left(\frac{1}{4}, \frac{1}{4}, \frac{1}{4}\right)$ and $48(\mathrm{i})$ at $\left(x, x, \frac{1}{2}\right)$ with $\frac{1}{3}<x<\frac{1}{2}$ sites (illustrating cation disorder in the $\langle 110\rangle$ direction). All these models were either unstable in the refinement due to high correlations between the fitted parameters or produced a worse fit to the experimental data.

The model with random occupation of the tetrahedral $8(\mathrm{c})$ site at $\left(\frac{1}{4}, \frac{1}{4}, \frac{1}{4}\right)$ and the octahedral 32(f) site at $(x, x, x)$ with $\frac{1}{3}<x<\frac{1}{2}$ yields the following temperature dependence 


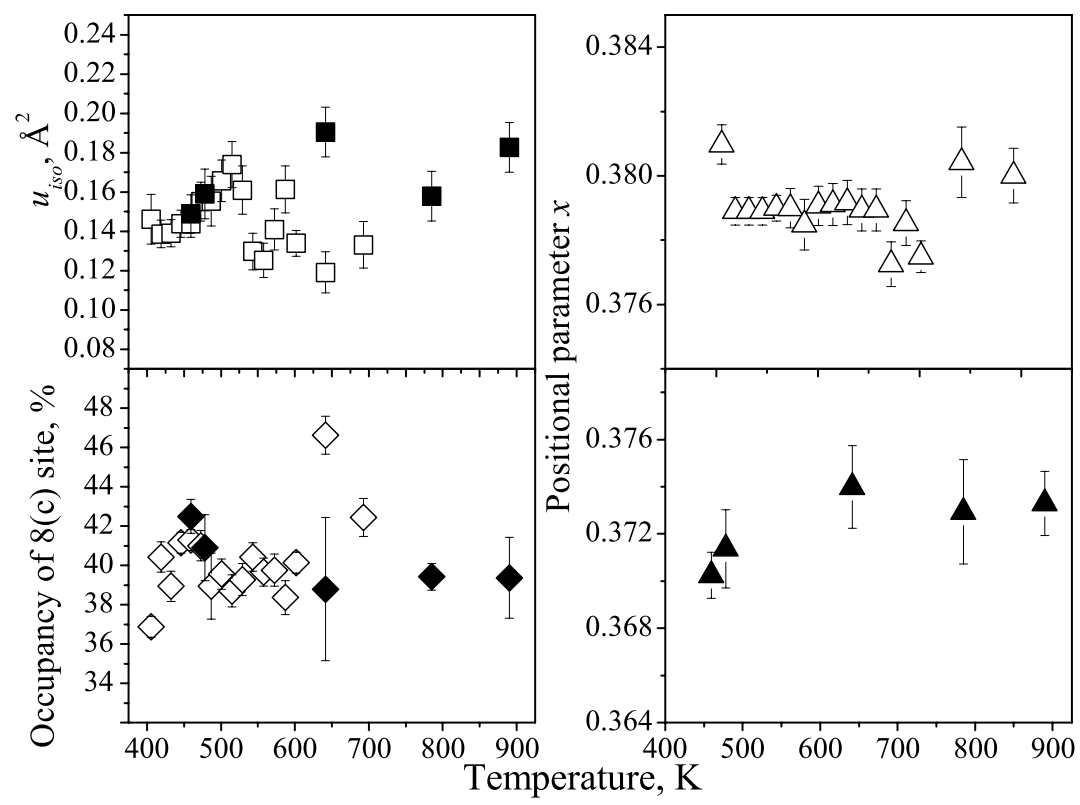

Figure 6. Temperature dependences of overall thermal parameters, normalized site occupation number of $\mathrm{Ag}^{+} / \mathrm{Cu}^{+}$residing on the tetrahedrally coordinated $8(\mathrm{c})$ sites and cation positional parameter of 32(f) at $(x, x, x)$ sites within the cubic $\delta$-AgCuS in the framework of model 5 from tables 2 and 3. Open symbols—-synchrotron data (B2), closed symbols—neutron data (SPODI).

for the parameters of the average structure: overall temperature factor, $u_{\mathrm{ov}}$, practically does not change with temperature; the cation positions of the octahedral site $32(\mathrm{f})$ at $(x, x, x)$ with $\frac{1}{3}<x<\frac{1}{2}$ do not change with temperature; there are no pronounced changes in the temperature dependence of the cation occupancies (see figure 6). Within this model, disorder occurs in $\langle 111\rangle$ directions towards the faces of octahedra rather than to their edges; in spite of the significant octahedral occupation there is only a minimal cation density on the ideal octahedral site 4(b) at $\left(\frac{1}{2}, \frac{1}{2}, \frac{1}{2}\right)$. In the light of these structural features, it is likely that cations jump in skewed $\langle 100\rangle$ directions between nearest-neighbour tetrahedral sites via the peripheries of the octahedral cavities. The same mechanism of ionic conductivity was proposed on the basis of powder diffraction studies for the superionic antifluorite phases of $\mathrm{Ag}_{2} \mathrm{Te}$ [18], $\mathrm{Cu}_{2-\delta} \mathrm{Se}$ $(\delta=0,0.15,0.25)$ [19] and $\mathrm{AgCuSe}[20]$. Note that diffraction results on a number of copperand silver based chalcogenides and halides (for instance, [19-22]) imply that ionic conductivity can be sensitive to details of the cation redistribution between available interstitial sites versus temperature as well as to the stoichiometry of the material. Taking this into account, we can propose a correlation between the results of the electrochemical measurements from Kadrgulov et al [3], which revealed only a relatively modest increase in the ionic conductivity with temperature within $\delta$ - $\mathrm{AgCuS}$, and our diffraction results, which show no pronounced cation redistribution. The same correlation was reported for superionic fcc-AgCuSe [20] as well as for superionic fcc- $\mathrm{Ag}_{x} \mathrm{~Pb}_{1-x} \mathrm{I}_{2-x}$ with $x=\frac{1}{3}$ and $\frac{2}{3}$ [21].

3.2.3. Thermal expansion. For convenience, the unit-cell parameters $a_{\mathrm{or}}, b_{\mathrm{or}}, c_{\mathrm{or}}$ of the orthorhombic $\mathrm{C}$-centred $\beta$-AgCuS were transformed into the primitive pseudo-hexagonal cell with dimensions $a_{\mathrm{phex}}, c_{\mathrm{phex}}, \gamma_{\mathrm{phex}}$ via $a_{\mathrm{phex}}=b_{\mathrm{phex}}=\sqrt{a_{\mathrm{or}}^{2}+b_{\mathrm{or}}^{2}} / 2, c_{\mathrm{phex}}=c_{\mathrm{or}}$ and $\gamma_{\text {phex }}=\arccos \left[\left(a_{\text {or }}^{2}-b_{\text {or }}^{2}\right) /\left(a_{\text {or }}^{2}+b_{\text {or }}^{2}\right)\right]$. The unit-cell parameter of cubic fcc $\delta$-AgCuS was 


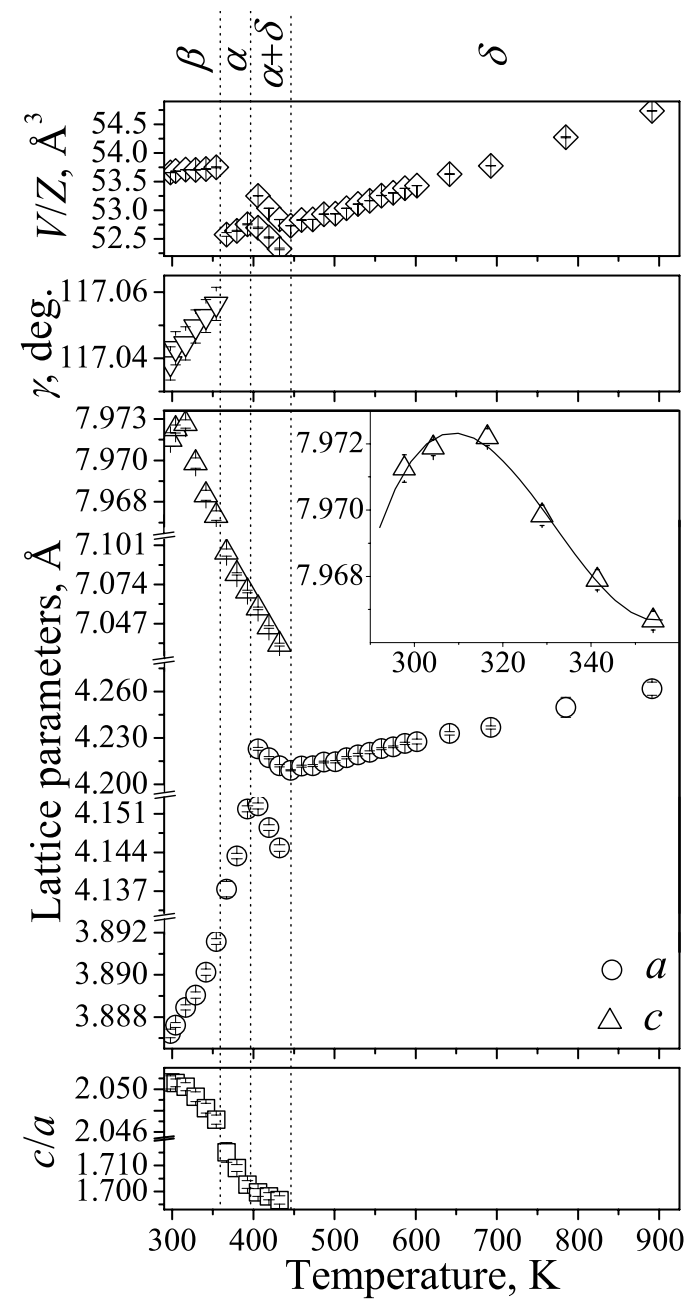

Figure 7. The unit cell axial ratio $c / a$, cell dimensions $a, c, \gamma$ and unit cell volume per formula unit $V / Z$ of stromeyerite as a function of temperature in the range from RT up to melting point. The lattice parameters of the primitive pseudo-hexagonal and rhombohedral cells of $\beta$ - and $\delta$-AgCuS are illustrated. Inset: unusual thermal expansion along the $c$-direction of $\beta$-AgCuS.

transformed into the primitive rhombohedral one via $a_{\mathrm{rh}}=\sqrt{2} a_{\mathrm{c}} / 2$, where $a_{\mathrm{rh}}$ and $a_{\mathrm{c}}$ are the cell dimensions of the rhombohedral and cubic cells, respectively.

The dimensions of the primitive cell, cell axial ratio and cell volume per formula unit of stromeyerite as a function of temperature are illustrated in figure 7. Analysis of the thermal behaviour of the lattice parameters revealed strong anisotropy of the thermal expansion in the $\beta$ - and $\alpha$-AgCuS. Negative thermal expansion in the $c$-direction was observed in these phases between 316 and $440 \mathrm{~K}$. Furthermore, the thermal expansion in $\beta$-AgCuS along the $c$-direction changing from positive at RT to a negative value above $316 \mathrm{~K}$ should be noted (see the inset in figure 7). No anomalies in the thermal expansion in the $a$-direction were revealed in the range RT-399 K. On entering the $\alpha+\delta$ region above $399 \mathrm{~K}$ the thermal expansion of the $\alpha$-AgCuS in the $a$-direction suddenly reverses sign and remains negative up to $439 \mathrm{~K}$. Similarly, the thermal expansion coefficient of the $\delta$ - $\mathrm{AgCuS}$ is negative in the $\alpha+\delta$ region, but positive in the 
pure $\delta$ phase. Additionally, the axial $c / a$ ratio in pseudo-hexagonal $\beta$-AgCuS and hexagonal $\alpha$ $\mathrm{AgCuS}$ acting as a measure for the deviation from an ideal hep structure shows a reduction with increasing temperature towards the close-packed 'ideal' value of 1.633. Hence, the hexagonal distortion in both $\beta$ - and $\alpha$-phases decreases on increasing the temperature. The drop in the $c / a$-value is drastically slowed in the mixed $\alpha+\delta$ region. The negative thermal expansion along the $c$-axis accompanied by a decrease in the $c / a$ ratio towards the 'ideal' value was also reported for other superionic compounds (see, for instance, hexagonal $\beta$-CuI $[23,24]$ ). The discontinuous volume changes (see figure 7) of about $2.3 \%$ and $0.6 \%$ at the $\beta \longrightarrow \alpha$ and $\alpha \longrightarrow \delta$ transformations are a very strong indication for a first order phase transition. The temperature dependence of the volume thermal expansion was fitted by linear functions and the volume thermal expansion coefficients within single-phase regions are $26 \times 10^{-6}, 130 \times 10^{-6}$ and $85 \times 10^{-6} \mathrm{~K}^{-1}$ for the $\beta$-, $\alpha$ - and $\delta$-phases, respectively. A negative expansion of the cell volumes of hexagonal and cubic stromeyerite was revealed in the $\alpha+\delta$ region. An extrapolation of the cell volumes of the pure $\alpha$ - and $\delta$-phases into $\alpha+\delta$ region gives $\alpha$-phase exhibiting a higher volume than the $\delta$-phase, but throughout this region the experimentally observed cell volume of $\alpha-\mathrm{AgCuS}$ is lower than that of the $\delta$-phase. The origin of this unusual thermal expansion observed in the range between 399 and $439 \mathrm{~K}$ is not yet finally understood. We can only assume that such a 'striking' behaviour is associated with the drastic rearrangement of the rigid anion framework occurring upon the hcp $\longrightarrow$ fcc transformation.

\section{Summary}

Large volume changes $(2.3 \%$ and $0.6 \%)$ at abrupt superionic phase transitions are strong evidence that stromeyerite undergoes a type-I superionic transition according to the classification of Boyce and Huberman [25]. The presence of an hep sulfur sublattice in both $\beta$ - and $\alpha$-AgCuS implies that the $\beta \longrightarrow \alpha$ transformation is induced due to cation disordering effects (or clustering of mobile ions, which accelerates instability of the lowtemperature phase [26]), whereas a rearrangement of the rigid anion sublattice from hcp to fcc accompanies the $\alpha \longrightarrow \delta$ transformation. Hence, the superionic transitions in stromeyerite have a different origin. According to [15], there are relatively few examples of structural phase transitions between two superionic phases accompanied by a rearrangement of the rigid anion sublattice. A model for ionic conduction in $\alpha$ - and $\delta$-AgCuS was derived from powder diffraction data: similar to $\mathrm{Cu}_{2} \mathrm{~S}$ [16], the cation conductivity in $\alpha-\mathrm{AgCuS}$ is two dimensional and occurs in slabs perpendicular to the $c$-direction, whereas it is likely that cations jump in 'skewed' $\langle 100\rangle$ directions between nearest-neighbour tetrahedral sites via the peripheries of octahedral cavities in $\delta$-AgCuS. The principal common feature for diffusion in $\alpha$ - and $\delta$-AgCuS is no evidence for cation jumps through $\left(\frac{1}{2}, \frac{1}{2}, \frac{1}{2}\right)$ sites in the $\langle 111\rangle$ direction. Doubtless, single-crystal measurements of $\alpha$ - and $\delta$-AgCuS would provide more information about the underlying structure. Any 'single-crystal' studies are, however, practically impossible (or at least extremely difficult) even if single crystals are available, since the first-order superionic phase transition will lead to fracture. The temperature dependence of the cation redistribution in $\delta$ - $\mathrm{AgCuS}$ as described by our structural model correlates well with the temperature behaviour of the ionic conductivity and gives strong support to the validity of the model. Finally, experimental studies by total scattering (Bragg and diffuse) on powdered samples or molecular dynamics simulations of diffusion pathways would provide further information for the ionic transport in stromeyerite. Hence, our powder diffraction investigation should be considered as a base for further investigations, either experimental or theoretical. 


\section{Acknowledgments}

Financial support from the Bundesministerium für Bildung und Forschung (grant number 03DU03D1) and from the Helmholtz Association of National Research Centres (grant number VH-VI 102) is gratefully acknowledged. We are also indebted to C Fasel for performing STA measurements, Dr A N Skomorokhov and Dr K G Bramnik for useful discussions.

\section{References}

[1] Hull S 2004 Rep. Prog. Phys. 67 1233-314

[2] Keen D A 2002 J. Phys.: Condens. Matter 14 R819-57

[3] Kadrgulov R F, Yakshibaev R A and Khasanov M A 2001 Ionics 7 156-60

[4] Suhr N 1955 Econ. Geol. 50 347-50

[5] Frueh A J 1955 Z. Kristallogr. 106 299-307

[6] Djurle S 1958 Acta Chem. Scand. 12 1427-36

[7] Skinner B J 1966 Econ. Geol. 61 1-26

[8] Skarda C, Wuensch B J and Prince E 1981 NBS Tech. Note 1160 57-63

[9] Baker C L, Lincoln F J and Johnson A W S 1991 Acta Crystallogr. B 47 891-9

[10] Knapp M, Baehtz C, Ehrenberg H and Fuess H 2004 J. Synchrotron Radiat. 11 328-34

[11] Pathak P D and Vasavada N G 1970 Acta Crystallogr. A 26 655-8

[12] Knapp M, Joco V, Baehtz C, Brecht H H, Berghaeuser A, Ehrenberg H, von Seggern H and Fuess H 2004 Nucl. Instrum. Methods A $\mathbf{5 2 1} 565-70$

[13] Hoelzel M, Senyshyn A, Gilles R, Boysen H and Fuess H 2006 Neutron News submitted

[14] http://www-llb.cea.fr/fullweb/powder.htm

[15] Hull S and Keen D A 2001 J. Phys.: Condens. Matter 13 5597-610

[16] Cava R J, Reidinger F and Wuensch B J 1981 Solid State Ion. 5 501-4

[17] Maichle J K, Ihringer J and Prandl W 1988 J. Appl. Crystallogr. 21 22-7

[18] Keen D A and Hull S 1998 J. Phys.: Condens. Matter 10 8217-34

[19] Skomorokhov A N, Trots D M, Knapp M, Bickulova N N and Fuess H 2006 J. Alloys Compounds 421 64-71

[20] Trots D M, Skomorokhov A N, Knapp M and Fuess H 2006 Eur. Phys. J. B 51 507-12

[21] Hull S, Keen D A and Berastegui P 2002 Solid State Ion. 147 97-106

[22] Keen D A, Hull S, Barnes A C, Berastegui P, Crichton W A, Madden P A, Tucker M G and Wilson M 2003 Phys. Rev. B 68014117

[23] Keen D A and Hull S 1994 J. Phys.: Condens. Matter 6 1637-44

[24] Keen D A and Hull S 1995 J. Phys.: Condens. Matter 7 5793-804

[25] Boyce J B and Huberman B A 1979 Phys. Rep. 51 189-265

[26] Ishii T 1998 Solid State Commun. 108 513-7 